Document downloaded from:

http://hdl.handle.net/10251/77962

This paper must be cited as:

Pons Tomás, P.; Jaén Martínez, FJ.; Catalá Bolós, A. (2015). Intelligent Playful Environments for Animals. ACM. doi:10.1145/2829875.2829879.

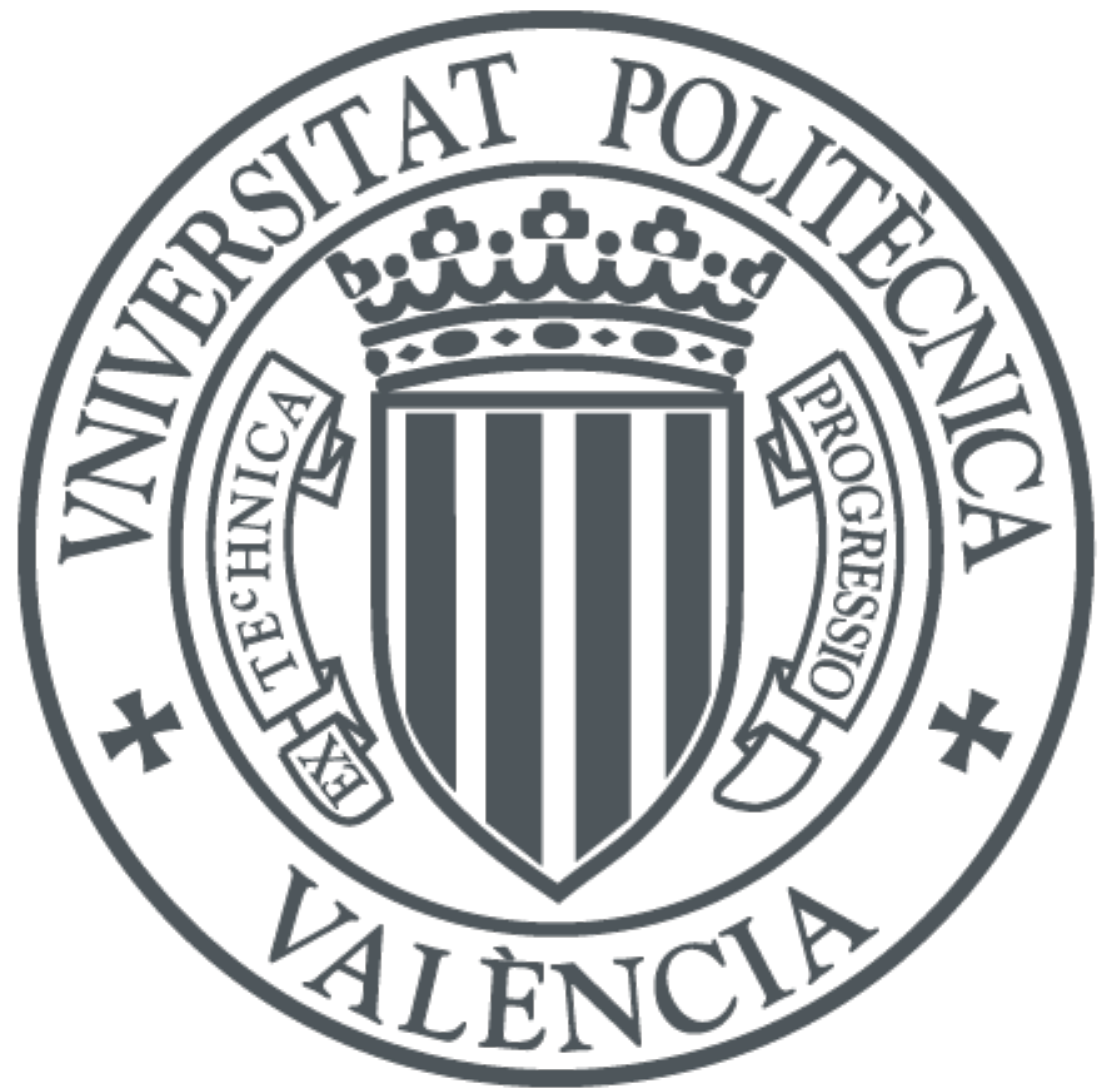

The final publication is available at

http://dl.acm.org/citation.cfm?doid=2829875.2829879

Copyright ACM

Additional Information

(C) Owner/Author 2015. This is the author's version of the work. It is posted here for your personal use. Not for redistribution. The definitive Version of Record was published in Interacción '15 Proceedings of the XVI International Conference on Human Computer Interaction, http://dx.doi.org/10.1145/2829875.2829879 


\title{
Intelligent Playful Environments for Animals
}

\author{
Patricia Pons, Javier Jaen, Alejandro Catala \\ Grupo ISSI, Departamento de Sistemas Informáticos y Computación, Universitat Politècnica de València \\ ppons@dsic.upv.es, fjaen@upv.es, acatala@dsic.upv.es
}

\begin{abstract}
We are evolving towards an interconnected and ubiquitous world, where digital devices and interfaces progressively adapt themselves to fit our needs and ease our daily activities. Although we coexist with plenty of animal species, such as our pets, we are approaching the evolution of technology in a strictly humancentric manner. A new field in Computer Science, called AnimalComputer Interaction (ACI), aims at filling this technological gap by developing systems and interfaces specifically designed for animals. Supporting animals' natural behavior and habits with suitable technology could improve both humans and animals' wellbeing. As a consequence, this doctoral research aims to explore, design and develop animal-centered intelligent systems that focus on enhancing one of the most natural animal behaviors: play. Therefore, the main goal of this research is to expand ACI with the ability of automatically manage and adapt animals play activity in order to improve their wellbeing.
\end{abstract}

\section{Categories and Subject Descriptors}

H.5.3 [Information Interfaces and Presentation]: User interfaces - interaction styles, user-centered design, input devices and strategies.

\section{General Terms}

Design, Experimentation.

\section{Keywords}

Animal Computer Interaction; Playful Interaction; Intelligent Environment; Animals; Interfaces.

\section{INTRODUCTION}

Human Computer Interaction (HCI) has brought countless benefits to human wellbeing. By easing the interaction with computer systems, technology has become more user-friendly and it is being applied to our everyday activities in a seamless manner. However, other species are not taking advantage of all these progresses. Due to different physical features or mental perceptions of the world, animals are not able to understand our technology as we do. Therefore, a new research field called Animal Computer Interaction (ACI) $[3,4]$ has emerged. It focuses on providing animals with similar technological improvements to the ones humans already have. ACI considers animals as the target users of digital systems, and proposes the development of interfaces specifically designed for them. The doctoral research presented in this paper proposes expanding ACI with the ability of automatically managing and adapting animals play activity in order to improve their wellbeing, focusing on animal pets and specifically in cats and dogs.

\section{RELATED WORKS}

There have been several research studies involving animals which use technology as a bridge to communicate or interact with them. The LANA Project [9], the Ai Project [5] or the SpeakDolphin [12] initiative are studies where the animals interact with specialized keyboards and/or touch screens to communicate with the trainers. Technology has also been used to improve critical tasks of some animals: ACI principles have been applied to develop suitable dog-oriented interfaces for Diabetes Alert Dogs (DAD), allowing them to alert emergency services when their owners suffer a hypoglycemic attack [8].

In recent years, several studies have focused on enhancing animal play using technology for different purposes. LonelyDog@Home [1] proposes a web interface where the human can connect to a web camera located at her home and check how their pets are, feed them or play with them remotely. Canine Amusement and Training [11] is a training system where, using a projector and cameras, the training activity becomes a playful experience. Felino [10] is a tabletbased game where a human and a cat can play together capturing different creatures on the screen. The human controls several aspects of the game, adapting it to the cat's reactions and preferences.

Although there are already games for animals which make use of technology to improve their experience, these games have some limitations which will be outlined in the next section.

\section{INTELLIGENT PLAYFUL ENVIRONMENTS FOR ANIMALS}

\subsection{Motivation}

The focus of this research activity on animal play is motivated by two reasons. On the one hand, play is one of the most natural behaviors among all animal species. Animals do not need to be taught on how to play [2], as it stands as a natural and voluntary activity, which has also its repercussion in elder stages of their lives. As an example, felines' play is always based on hunting or chasing behaviors, as in the wild, these animals traditionally used to hunt for food. On the other hand, verbal or written communication is unfeasible when considering animals as target users, and thus, other methods should emerge in order to determine the perceived usability and usefulness of the system. Therefore, playful interactions could be an effective way of communicating with animals and gathering information about the usefulness of a system. As summarized in Section 2, animal play has already started to be the object of a digital revolution. However, existing digital games for animals are focused on a single activity or purpose and only use one digital device. Moreover, they require human mediation for the animal to play 
and with time the animal might lose interest when it gets used to play the same game.

The main contribution of this doctoral research is two-fold. Firstly, we will consider digital games for animals as playful environments composed of several interconnected digital devices. Those devices have to be appealing and suitable for the animal and for the purpose of the game. Secondly, a playful environment of this characteristics will be an intelligent system which should intelligently make use of its composing devices in order to create suitable playful activities for different purposes. The intelligent environment should gather reliable information about the animal, its preferences, physical features and its surrounding context. With this knowledge, the intelligent environment should create an engaging playful activity for a specific situation, deciding which devices and interaction mechanisms would be more suitable for the given context. There are plenty of scenarios where intelligent playful environments could help to improve animals' wellbeing, e.g., create playful activities to alleviate the stress and anxiety of animals alone at home, zoos or shelters. It could also encourage these animals to perform physical exercise by means of play. The system should adapt the game to the animal's attributes such as species, weight or age - old animals should not perform as much physical exercise as young ones. An intelligent playful environment could also assist in animal training, making the activity more amusing and less repetitive, and without requiring human supervision. The environment could choose the optimal time of the day to perform training, i.e., when the animal's attention and motivation seems to be higher.

\subsection{Definition}

As part of the planning stage of the research, we have already defined the scope of the systems being developed $[6,7]$ :

An intelligent playful environment for animals, or IPE4A, is an animal-centered ecosystem with intelligent capabilities which is able to learn from the animals' behaviors and interactions, using the acquired knowledge to adapt itself to the context, creating engaging playful activities which do not necessarily need human mediation to evolve.

We have also outlined the different features and dimensions of intelligent playful environments which can affect the design and development of these systems [6,7]: number of participants, species of the participants, human participation, human presence, control, information acquisition, learning inputs, types of stimuli, purpose of the activity.

\subsection{Research questions}

This thesis will give answer to several fundamental questions on how to provide successful interactions and communication between the animal and the intelligent system. Some of these research questions are:

- Q1: Which are the most appropriate interactive mechanisms for the animal, e.g. a cat or a dog?

- Q2: How can the system gather information unobtrusively about the animals' preferences, actions and context?

- Q3: How can the system use all these information in order to adapt the playful activity to the context and the animals' preferences and wills?

- Q4: How can we objectively measure the success of the system?

\section{CONCLUSIONS AND FUTURE WORK}

This thesis proposes the definition, design and development of intelligent playful environments for animals, focusing on pet animals. These systems aim to automatically create and adapt playful digital experiences for animals, improving their wellbeing and providing a better understanding of their interactions with digital systems. We have already defined these environments, their features and potential [6,7]. Our immediate future work will be conducting a set of experiments in order to answer question Q1 in Section 3.3. For this purpose, we will study how different devices and stimuli affect the attention and motivation of the animal for starting a playful activity. Based on the results of these experiments, we will design suitable activities in order to conduct future studies to answer the remaining research questions.

\section{ACKNOWLEDGEMENTS}

Work supported by MINECO (TIN2010-20488 and TIN201460077-R), UPV (UPV-FE-2014-24), MECD (FPU13/03831) and GVA (APOSTD/2013/013).

\section{REFERENCES}

[1] Hu, F., Silver, D., and Trude, A.LonelyDog@Home. 2007 IEEE/WIC/ACM International Conferences on Web Intelligence and Intelligent Agent Technology - Workshops, IEEE (2007), 333-337.

[2] Huizinga, J.Homo ludens. Wolters-Noordhoff, Groningen, The Nederlands, 1985.

[3] Mancini, C.Animal-computer interaction: a manifesto. Magazine interactions 18, 4 (2011), 69-73.

[4] Mancini, C.Animal-computer interaction (ACI): changing perspective on HCI, participation and sustainability. CHI '13 Extended Abstracts on Human Factors in Computing Systems, ACM Press (2013), 2227-2236.

[5] Matsuzawa, T.The Ai project: historical and ecological contexts. Animal cognition 6, 4 (2003), 199-211.

[6] Pons, P., Jaen, J., and Catala, A.Animal Ludens : Building Intelligent Playful Environments for Animals. 11th Conference on Advances in Computer Entertainment Workshop on Animal Human Computer Interaction, (2014).

[7] Pons, P., Jaen, J., and Catala, A.Envisioning Future Playful Interactive Environments for Animals. In A. Nijholt, ed., More Playful User Interfaces. Springer, 2015.

[8] Robinson, C., Mancini, C., van der Linden, J., Guest, C., and Harris, R.Empowering assistance dogs : an alarm interface for canine use. Intelligent Systems for Animal Welfare, (2014).

[9] Rumbaugh, D.M., Gill, T. V., Brown, J. V., et al.A computer-controlled language training system for investigating the language skills of young apes. Behavior Research Methods \& Instrumentation 5, 5 (1973), 385-392.

[10] Westerlaken, M. and Gualeni, S.Felino: The Philosophical Practice of Making an Interspecies Videogame. The Philosophy of Computer Games Conference, (2014), 1-12.

[11] Wingrave, C.A., Rose, J., Langston, T., and LaViola, J.J.J.Early explorations of CAT: canine amusement and training. CHI '10 Extended Abstracts on Human Factors in Computing Systems, (2010), 2661-2669.

[12] SpeakDolphin. http://www.speakdolphin.com. 